\title{
Índice neutrófilo-linfocitario en la predicción de trombosis temprana del stent
}

\section{Neutrophil-lymphocyte index in the prediction of early stent thrombosis}

\author{
Suilbert Rodríguez Blanco, Abel Y. Leyva Quert, José M. Aguilar Medina
}

\section{RESUMEN}

Introducción. La inflamación es el principal mecanismo propuesto en la génesis fisiopatológica de la trombosis del stent. Una expresión del estado inflamatorio es el índice neutrófilo linfocitario. Objetivo. Evaluar el valor pronóstico del índice neutrófilo linfocitario en la predicción de trombosis temprana del stent. Método. Investigación prospectiva en el Hospital Hermanos Ameijeiras, La Habana, Cuba, entre diciembre de 2018 y junio 2019. Resultados. Se estudiaron 196 pacientes; las variables niveles elevados de neutrófilo $(p=0,009)$ e índice neutrófilo linfocitario $(p=0,007)$ después del proceder se relacionan significativamente con la presencia de trombosis del stent y la curva ROC encontró una sensibilidad de $70 \%$ y una especificidad de $62 \%$ para un nivel del índice mayor de 6 (0,712; IC95\%: 0,610-0,988; $p=0,012)$. Conclusión. El aumento del índice neutrófilo-linfocitario a las 6 horas del intervencionismo coronario percutáneo, tiene alta sensibilidad y alta especificidad en la predicción de trombosis temprana del stent.

Palabras clave: índice neutrófilo linfocitario, trombosis temprana del stent

\begin{abstract}
Introduction. Inflammation is the main mechanism proposed in the physiological genesis of stent thrombosis. An expression of the inflammatory state is the lymphocytic neutrophil index. Objective. Evaluate the prognostic value of the lymphococcal neutrophil index in the prediction of early thrombosis of the stent. Method. Prospective research at the Hermanos Ameijeiras Hospital, Havana, Cuba, between December 2018 and June 2019. Results. 196 patients were studied; the variables high levels of neutrophil ( $p-0.009)$ and lymphocytic neutrophil index ( $p-0.007)$ after the proceeding are significantly related to the presence of stent thrombosis and the ROC curve found a sensitivity of $70 \%$ and a specificity of $62 \%$ for an index level greater than $6(0.712$; IC95\%: 0.610-0.988; 0.012). Conclusion. The increase in the neutrophil-lymphocytic index at 6 hours of percutaneous coronary interventionism has high sensitivity and high specificity as predictive value in early stent thrombosis
\end{abstract}

Keywords: lymphocytic neutrophil index, early stent thrombosis.

Revista Argentina de Cardioangiología Intervencionista 2020;11(1):17-20. https://doi.org/10.30567/RAC1/202001/0017-0020

\section{INTRODUCCIÓN}

La trombosis del stent (TS) es una de las principales complicaciones del intervencionismo coronario percutáneo (ICP) y se asocia con altas tasas de morbilidad y mortalidad ${ }^{1-3}$. Es un proceso complejo y multifactorial donde se han descrito factores relacionados con el paciente: interrupción temprana de la terapia de doble antiagregación plaquetaria, la diabetes mellitus, paciente inestable, el antecedente de TS previa y otros; factores anatómicos: la extensión de la enfermedad coronaria, vasos finos, lesiones ostiales, bifurcaciones coronarias y otros y factores relacionados con el proceder: técnica compleja en bifurcación, longitud del segmento tratado y otros ${ }^{2}$.

En 2008 se publicaron las pautas del Consorcio de Investigación Académica con respecto a las clasificaciones de la $\mathrm{TS}^{4}$. Según el tiempo de aparición esta se clasifica en aguda, subaguda, tardía y muy tardía. La aguda (menos de 24 horas) y la subaguda (desde las 24 horas y hasta 30 días) se denominan trombosis temprana del stent. La TS probable se define como cualquier muerte inexplicable en los primeros 30 días después de la colocación del stent o la presencia de síndrome coronario agudo con elevación del segmento ST con topografía concordante con el territorio de la arteria tratada. Por su parte, la TS definitiva requiere la confirmación angio-

1. Departamento de cardiología intervencionista. Hospital Hermanos Ameijeiras, La Habana, Cuba

$\bowtie$ Correspondencia: Suilbert Rodríguez Blanco. suilbertr@infomed.sld.cu; suilbertrb@gmail.com

Los autores no declaran conflictos de intereses

Recibido: 11/02/2019| Aceptado: 24/12/2019 gráfica o anatomopatológica de la presencia de trombo dentro del stent o en los 5 milímetros anterior o posterior a este. Los avances en el tratamiento antiplaquetario, las continuas mejoras de los stents y de la técnica de implante han disminuido la incidencia a $0,7 \%$. Esta complicación es menor en la intervención electiva $(0,3$ a $0,5 \%)$ que en la urgencia $(3,4 \%)^{5,6}$.

Los stents son prótesis endovasculares potencialmente trombogénicas, propiedad que depende ampliamente de la carga electropositiva de la superficie metálica que parece estar modificada por la composición, configuración y tamaño del mismo y consecuentemente a la respuesta protectora del vaso. Más allá de las propiedades trombogénicas del stent en su condición de estructura metálica, después de su implante, son los factores clínicos, técnicos y del procedimiento los que juegan un papel determinante en la ocurrencia de TS aguda y/o subaguda (TS temprana); no así cuando los eventos trombóticos se consideran tardíos, los cuales parecen estar directamente relacionados a una compleja respuesta mediata y tardía del vaso con respecto a la presencia del stent (generalmente farmacológico), la plataforma, la droga y el polímero. Esta respuesta está dada por: endotelización incompleta y formación neointimal, hipersensibilidad, disfunción endotelial (respuesta inflamatoria tardía) y aposición incompleta tardía y adquirida del stent $t^{7-9}$.

La tasa de TS temprana parece ser algo mayor con stents metálicos que con los farmacoactivos. Se ha sugerido que la matriz polimérica de los stent farmacoactivos puede reducir la trombogenicidad aguda, y los factores relacionados con el procedimiento son los más importantes (mala aposición del stent, infraexpansión, disección de bordes) aunque también intervienen factores clínicos como la diabetes mellitus, FEVI reducida, rasgos genéticos, entre otros, que confieren un estado inflamatorio ${ }^{10,11}$. 


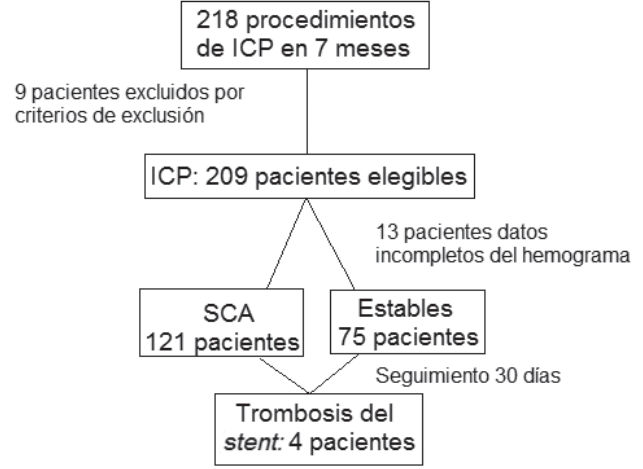

Figura 1. Flujograma de la muestra estudiada.

Este factor inflamación, descrito en la TS tardía y muy tardía, se relaciona con la implantación del stent y existe evidencia que relaciona marcadores inflamatorios y $\mathrm{TS}^{12}$.

El índice neutrófilo linfocitario (INL) es un marcador inflamatorio que se relaciona con la presencia, gravedad y extensión de la enfermedad coronaria ${ }^{13}$, con eventos cardiovasculares adversos en pacientes con infarto agudo tratado mediante intervencionismo ${ }^{14}$, y con la presencia de infarto del miocardio periprocedimiento tipo $4 \mathrm{a}^{15}$.

Este biomarcador INL, de fácil determinación, disponible, reproducible y económico, emerge como marcador del estado inflamatorio. En este sentido, existen antecedentes que relacionan el INL con la TS y la alta mortalidad en pacientes con síndrome coronario agudo ${ }^{16}$.

\section{OBJETIVO}

Evaluar el valor pronóstico del índice neutrófilo linfocitario en la predicción de trombosis temprana del stent.

\section{DISEÑO METODOLÓGICO}

Tipo de investigación: aplicada, descriptiva-correlacional y prospectiva.

Período y lugar: Hospital Hermanos Ameijeiras, La Habana, Cuba, entre diciembre de 2018 y junio 2019.

\section{PARTICIPANTES}

\section{Criterios de inclusión}

- Pacientes mayores de 18 años a quienes se realice intervencionismo coronario percutáneo.

\section{Criterios de exclusión}

- Disfunción sistólica severa del ventrículo izquierdo (fracción de eyección <30\%).

- ICP sobre oclusión total crónica.

- Cirugía de revascularización miocárdica en los 3 meses anteriores.

- Enfermedad pulmonar grave. Neoplasia. Desorden hematológico crónico.

- Terapia con corticoesteroides en el último año. Enfermedad inflamatoria crónica. Infección activa al momento de la intervención.

\section{Muestra}

Conformada por 196 pacientes de forma consecutiva que cumplieron los criterios de inclusión y exclusión en el período señalado.

\section{Variable principal}

- Índice neutrófilo- linfocitario: Se definió como el cociente entre el valor absoluto de neutrófilos y el valor absoluto de linfocitos. Éste se determinó previo al proceder intervencionista y a las 6 horas luego de realizado este.

- Trombosis del stent: Se definió como TS temprana (probable o definitiva) la ocurrida en los primeros 30 días después del implante del stent.

\section{Técnicas y procedimientos}

- Análisis automático del leucograma: se realizó en un equipo de hemograma automatizado Pentra-DX NEXUS. Analizador hematológico completamente automatizado para el diagnóstico in-vitro de muestras de sangre total anticoagulada con EDTA al 10\%.

- Linfocitos: [LYN], expresado en valor absoluto (\#).

- Neutrófilos: [NEU], expresado en valor absoluto (\#)

\section{Estadísticas}

La comparación de valores no peramétricos entre ambos grupos se realizó con la prueba de la U de Mann-Whitney. Las variables categóricas se compararon con Chi cuadrado. El análisis de regresión se utilizó para evaluar los predictores de trombosis del stent. Aquellas variables con $\mathrm{p}<0,05$ por análisis univariado fueron incluidas en un modelo de análisis de regresión multivariado, calculándose sus odds ratio (OR) con intervalo de confianza del 95\% (IC95\%). La curva ROC se construyó para determinar la predicción del valor de INL en la trombosis del stent.

\section{RESULTADOS}

El flujograma de la Figura 1 muestra que en el período estudiado se realizaron 218 intervenciones. De los 209 pacientes elegibles, 13 tenían datos incompletos y se estudiaron 196 pacientes, de los cuales 4 presentaron trombosis temprana del stent $(2,04 \%)$.

Las variables clínicas, angiográficas, relacionadas con el proceder y de laboratorio se recogen en la Tabla 1. La distribución de estas según la presencia o no de trombosis temprana del stent muestra que variables como edad avanza$\mathrm{da}(\mathrm{p}=0,047)$, presencia de diabetes mellitus $(\mathrm{p}=0,04)$, lesión en bifurcación $(\mathrm{p}=0,036)$ y síndrome coronario agudo $(\mathrm{p}=0,04)$ se asocian a la presencia de esta complicación. De la misma forma, los niveles elevados de neutrófilo $(\mathrm{p}=0,009)$ y de INL $(p=0,007)$ después del proceder se relacionan significativamente con la presencia de TS.

La distribución de los pacientes con TS según tiempo de aparición, diagnóstico definitivo y localización no muestra significación estadística (Tabla 2), resultado influido por la poca frecuencia de trombosis del stent.

El nivel del INL según la presencia o no de TS se muestra en las Figuras 2 y 3. En los pacientes con trombosis temprana del stent el INL después del proceder se encontró elevado $(\mathrm{p}<0,05)$ y la curva ROC muestra una sensibilidad de $70 \%$ y una especificidad de $62 \%$ para un nivel de INL mayor de 6 (0,712; IC95\%: 0,610-0,988; $\mathrm{p}=0,012)$. 
TABLA 1. Variables clínicas, angiográficas, dependientes del proceder y parámetros de laboratorio en pacientes estudiados.

\begin{tabular}{|c|c|c|c|c|}
\hline \multirow[t]{2}{*}{ Variable } & \multirow[t]{2}{*}{ Pacientes } & \multicolumn{2}{|c|}{$\begin{array}{l}\text { Presencia de trombosis } \\
\text { temprana del stent }\end{array}$} & \multirow[t]{2}{*}{$p$} \\
\hline & & Sí (4) & No (192) & \\
\hline Edad & $65,3 \pm 9,1$ & $69,6 \pm 8,2$ & $63,1 \pm 6,1$ & 0,047 \\
\hline Hombre (\%) & $122(62,2 \%)$ & $3(75 \%)$ & $119(61,9 \%)$ & 0,23 \\
\hline DM (\%) & $83(42,3 \%)$ & $4(100 \%)$ & $79(41,1 \%)$ & 0,04 \\
\hline Tabaquismo & $92(46,9 \%)$ & $2(50 \%)$ & $90(46,9 \%)$ & 0,6 \\
\hline$E R C>1(\%)$ & $31(15,8 \%)$ & $1(25 \%)$ & $30(15,6 \%)$ & 0,78 \\
\hline IM previo & $52(26,5 \%)$ & $2(50 \%)$ & $50(26,0 \%)$ & 0,09 \\
\hline FEVI & $55,4 \pm 3$ & $46 \pm 8,9$ & $57 \pm 4,2$ & 0,056 \\
\hline Al/IMASEST & $121(38,2 \%)$ & $3(75 \%)$ & $118(61,5 \%)$ & 0,04 \\
\hline ASA + clopidogrel & $196(100 \%)$ & $4(100 \%)$ & $192(100 \%)$ & 1 \\
\hline Estatinas & $133(67,9 \%)$ & $4(100 \%)$ & $129(67,2 \%)$ & 0,12 \\
\hline Score SYNTAX & $24,1 \pm 4,2$ & $28,2 \pm 4,1$ & $23,0 \pm 3,3$ & 0,23 \\
\hline Lesión en bifurcación (\%) & $71(36,2 \%)$ & $4(100 \%)$ & $67(34,9 \%)$ & 0,036 \\
\hline $\begin{array}{l}\text { Longitud total del } \\
\text { segmento stentado (mm) }\end{array}$ & $21 \pm 9,2$ & $28 \pm 10,1$ & $20 \pm 8,3$ & 0,38 \\
\hline Presencia de trombo & $12(6,12 \%)$ & $1(25 \%)$ & $11(5,7 \%)$ & 0,09 \\
\hline \multicolumn{5}{|c|}{ Arteria relacionada con la trombosis del stent } \\
\hline $\mathrm{TCl}$ & $18(9,2 \%)$ & $1(25 \%)$ & $17(8,9 \%)$ & 0,9 \\
\hline DA & $88(44,9 \%)$ & $2(50 \%)$ & $86(44,8 \%)$ & 0,45 \\
\hline Diagonal & $6(3,1 \%)$ & $1(25 \%)$ & $5(2,6 \%)$ & 0,2 \\
\hline$C x$ & $29(14,8 \%)$ & 0 & $29(15,1 \%)$ & \\
\hline$C D$ & $79(40,3 \%)$ & 0 & $79(41,1 \%)$ & \\
\hline $\begin{array}{l}\text { Técnica compleja } \\
\text { en bifurcación }\end{array}$ & $4(2 \%)$ & 0 & $4(2,1 \%)$ & \\
\hline Stent convencional & $109(55,6 \%)$ & $1(25 \%)$ & $108(56,3 \%)$ & 0,6 \\
\hline Stent fármacoactivo & $87(44,4 \%)$ & $3(75 \%)$ & $84(43,8 \%)$ & 0,5 \\
\hline $\begin{array}{l}\text { Neutrófilos }\left(\times 10^{3} / \mathrm{mm}^{3}\right) \\
- \text { antes de proceder } \\
\text { - después de proceder }\end{array}$ & $\begin{array}{l}3,0 \pm 1,2 \\
3,8 \pm 1,8\end{array}$ & $\begin{array}{l}3,4 \pm 0,5 \\
5,8 \pm 0,4\end{array}$ & $\begin{array}{l}3,0 \pm 1,1 \\
3,7 \pm 1,2\end{array}$ & $\begin{array}{r}0,08 \\
0,009\end{array}$ \\
\hline $\begin{array}{l}\text { Linfocitos }\left(\times 10^{3} / \mathrm{mm}^{3}\right) \\
\text { - antes de proceder } \\
\text { - después de proceder }\end{array}$ & $\begin{array}{l}1,9 \pm 1,3 \\
1,4 \pm 1,2 \\
\end{array}$ & $\begin{array}{l}1,9 \pm 1,4 \\
0,9 \pm 0,4 \\
\end{array}$ & $\begin{array}{l}2,0 \pm 1,0 \\
1,9 \pm 1,2\end{array}$ & $\begin{array}{l}0,08 \\
0,14\end{array}$ \\
\hline $\begin{array}{l}\text { INL } \\
\text { - antes de proceder } \\
\text { - después de proceder }\end{array}$ & $\begin{array}{l}2,1 \pm 1,5 \\
2,7 \pm 1,8\end{array}$ & $\begin{array}{l}3,5 \pm 1,3 \\
6,5 \pm 0,3\end{array}$ & $\begin{array}{l}2,8 \pm 1,9 \\
3,0 \pm 1,0\end{array}$ & $\begin{array}{c}0,09 \\
0,007\end{array}$ \\
\hline
\end{tabular}

DM: diabetes mellitus. IM: infarto de miocardio. FEVI: fracción de eyección del ventrículo izquierdo. Al: angina inestable. IMASEST: infarto miocárdico agudo sin elevación del segmento ST. ASA: ácido acetilsalicílico. INL: índice neutrófilo linfocitario.

TABLA 2. Distribución de los pacientes con trombosis del stent temprana se gún tiempo y localización.

\begin{tabular}{|l|c|c|c|c|c|c|c|}
\hline & $\begin{array}{c}\text { TS } \\
\text { aguda }\end{array}$ & $\begin{array}{c}\text { TS } \\
\text { subaguda }\end{array}$ & $\begin{array}{c}\text { TS } \\
\text { definitiva }\end{array}$ & $\begin{array}{c}\text { TS } \\
\text { probable }\end{array}$ & TCI & DA & Diag. \\
\hline Caso 1 & $X$ & & $X$ & & & $X$ & \\
\hline Caso 2 & & $X$ & $X$ & & & $X$ & \\
\hline Caso 3 & & $X$ & $X$ & & & & $X$ \\
\hline Caso 4 & & $X$ & & $X$ & $X$ & & \\
\hline
\end{tabular}

TS: trombosis del stent.

En el análisis multivariado, solo las variables lesión en bifurcación (0,905; IC95\%: 0,744-0,991; $\mathrm{p}=0,003)$, niveles elevados de neutrófilos después del proceder $(0,833$; IC95\%: 0,645$0,911 ; \mathrm{p}=0,012)$ y del INL después del proceder $(0,809$; IC95\%: 0,689-0,873; $p=0,009)$ se asociaron de forma independientes con la presencia de esta complicación (Tabla 3).

\section{DISCUSIÓN}

La inflamación es el principal mecanismo propuesto en la génesis fisiopatológica de la trombosis del stent ${ }^{12}$. Una expresión del estado inflamatorio presente en las arterias coronarias es el índice neutrófilo linfocitario. El aumento de neutrófilos incrementa la secreción de marcadores inflamatorios como la enzima pro-
TABLA 3. Análisis multivariado de variables relacionadas con la presencia de trombosis del stent.

\begin{tabular}{|l|c|c|}
\hline Variables & OR (IC95\%) & $\mathrm{P}$ \\
\hline Edad & $1,023(0,874-2,064)$ & 0,147 \\
\hline Diabetes mellitus & $0,994(0,836-1,582)$ & 0,245 \\
\hline Al/IMASEST & $1,693(0,726-2,059)$ & 0,133 \\
\hline Lesión en bifurcación & $0,905(0,744-0,991)$ & 0,003 \\
\hline Neutrófilos después del proceder (6 horas) & $0,833(0,645-0,911)$ & 0,012 \\
\hline INL antes del proceder & $1,19(0,428-1,977)$ & 0,544 \\
\hline INL después del proceder (6 horas) & $0,809(0,689-0,873)$ & 0,009 \\
\hline
\end{tabular}

OR: odds ratio. IC95\%: intervalo de confianza del 95\%. TS: trombosis del stent. Al: angina inestable. IMASEST: infarto miocárdico agudo sin elevación del segmento ST.

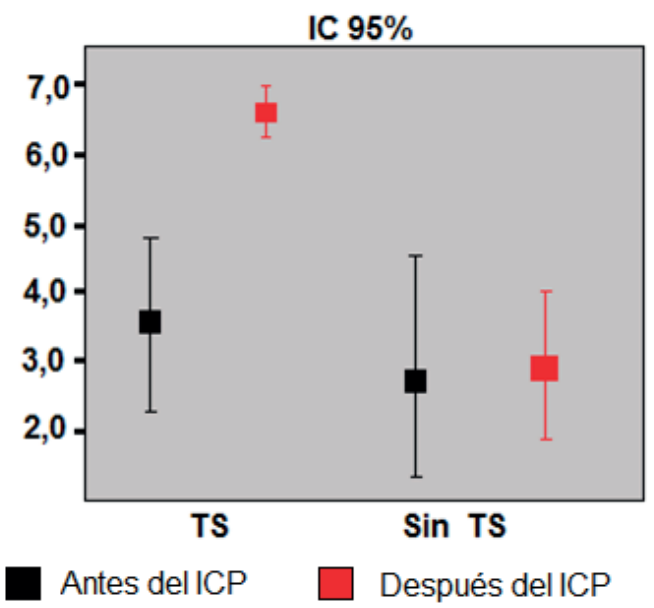

Figura 2. Relación del INL según la presencia o no de TS.

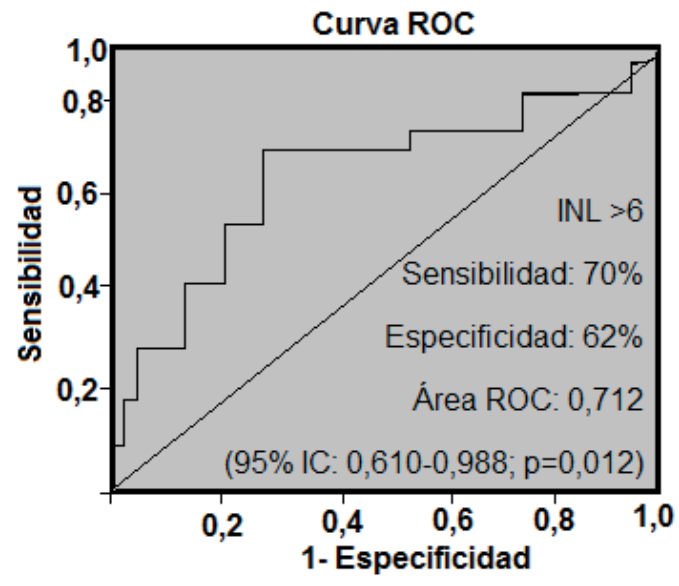

Figura 3. Curva ROC. Indice neutrófilo linfocitario en la predicción de TS.

teolítica elastasa, que produce degradación de la membrana basal en la pared vascular y daño endotelial ${ }^{17}$. En contraste, existe una disminución de los niveles de linfocitos asociados a la secreción de cortisol, que empeora la respuesta inflamatoria ${ }^{18}$.

Nuestros resultados indican una asociación significativa entre los niveles aumentados de INL mayores de 6 después del proceder y la presencia de trombosis temprana del stent, con una sensibilidad y especificidad alta.

Resultados similares encontraron Burak A et al. ${ }^{16}$, que demostraron que un INL mayor de 4,9 tiene alta sensibilidad y especificidad en la predicción de mortalidad intrahospitalaria y relación significativa con la presencia de TS.

De manera independiente, el conteo de neutrófilos a las 6 horas del proceder se asoció a la presencia de trombosis del stent 
en la presente serie. Estudios previos relacionan este parámetro con el aumento de la mortalidad cardiovascular por enfermedad coronaria ${ }^{19}$, lo que explica el estado inflamatorio presente en el árbol coronario en pacientes que presentan TS.

\section{LIMITACIÓN DEL ESTUDIO}

Este es un estudio con escaso tamaño de muestra. Aunque el porcentaje de pacientes con trombosis temprana del stent es

\section{BIBLIOGRAFÍA}

1. Longobardo L, Mattesini A, Valente S, Di Mario C. OCT-guided Percutaneous Coronary Intervention in Bifurcation Lesions. Interv Cardiol 2019 Feb; 14(1):5-9.

2. Piranavan P, KaurNJ,Marmoush F, BurtonA, Hannan J. Ticagrelor-induced Angioedema After Percutaneous Coronary Intervention in a Patient with a History of Ischemic Stroke and Low Response to Clopidogrel: A Rare Dilemma. Cureus. 2018 Dec 11;10(12):e3720.

3. Lee SN, Moon D, Moon KW, Yoo KD. The Glasgow prognostic score as a significant predictor of clinical outcomes in patients with acute coronary syndrome. J Cardiol 2019;74(2):130-5.

4. Applegate R, Sacrinty M, Little W, Santos R, Gandhi S, Kutcher M. Incidence of Coronary stent Thrombosis Based on Academic Reserch Consortium Definitions 2008;102(16):683-8.

5. Kim YH, Her AY, Jeong MH, Kim BK, Hong SJ, Ahn CM, et al. A comparison of the impact of current smoking on 2-year major clinical outcomes of first-and second-generation drug-eluting stents in acute myocardial infarction: Data from the Korea Acute Myocardial Infarction Registry. Medicine (Baltimore). 2019;98(10):e14797.

6. Zhao XY, Li JX, Tang XF, XU JJ, Song Y, Jiang L, et al. Validation of Predictive Value of Patterns of Nonadherence to Antiplatelet Regimen in Stented Patients Thrombotic Risk Score in Chinese Population Undergoing Percutaneous Coronary Intervention: A Prospective Observational Study. Chin Med J 2018;20;131(22):2699-704.

7. Byrne RA, Joner M, Kastrati A. Stent thrombosis and restenosis: what have we learned and where are we going? The Andreas Grüntzig Lecture ESC 2014. Eur Heart J. 2015 Dec 14;36 (47):3320-31.

8. Nakano M, Yahagi K, Otsuka F, et al. Causes of early stent thrombosis in patients presenting with acute coronary syndrome: an exvivo human autopsy study. J Am Coll Cardiol. 2014;63:2510-20.

9. Lee S, Ahn J, Mintz GS, et al. Characteristics of Earlier Versus Delayed Presentationof Very Late Drug-Eluting Stent Thrombosis: An Optical Coherence Tomographic Study. J Am Heart Assoc 2017;6:e005386. comparable al reportado en la literatura, estos pacientes están poco representados en el estudio.

\section{CONCLUSIÓN}

El aumento del índice neutrófilo linfocitario a las 6 horas del intervencionismo coronario percutáneo tiene alta sensibilidad y alta especificidad en la predicción de trombosis temprana del stent.

10. Cuesta J, Rivero F, Bastante T. Optical Coherence Tomography Findings in Patients With Stent Thrombosis. Rev Esp Cardiol. 2017;70:1050-8.

11. Souteyrand G, Amabile N, Mangin L. Mechanisms of stent thrombosis anlysed by optical coherence tomography: insights from the national PESTO French registry. Eur Heart J. 2016;37:1208-16.

12. Niccoli G, Montone RA, Ferrante G, Crea F. The evolving role of inflammatory biomarkers in risk assessment after stent implantation. J Am Coll Cardiol 2010;56(22):1783-93.

13. Açar G, Fidan S, Uslu ZA, Turkday S, Avci A, Alizade E, et al. Relationship of neutrophil-lymphocyte ratio with the presence, severity, and extent of coronary atherosclerosis detected by coronary computed tomography angiography. Angiology 2015;66(2):174-9.

14. Sai Zhang, Jun Diao, Chunmei Qi, Jingjing Jin, Li Li, XingjuanGao, et al. Predictive value of neutrophil to lymphocyte ratio in patients with acute ST segment elevation myocardial infarction after percutaneous coronary intervention: a meta-analysis. BMC Cardiovascular Disorders 2018,8:75.

15. https://doi.org/10.1186/s12872-018-0812-6

16. Bressi E, Mangiacapra F, Ricottini E, Cavallari l, Colaiori I, Di Gioia G, et al. Relation of Neutrophil to Lymphocyte Ratio With Periprocedural Myocardial Damage in Patients Undergoing Elective Percutaneous Coronary Intervention. Am J Cardiol 2016;118:980-4

17. Burak Ayca, Fatih Akın, Omer Celik, Irfan Sahin, S. Sezai Yildiz, I. Ilker Avci, et al. Neutrophil to Lymphocyte Ratio is Related to Stent Thrombosis and High Mortality in Patients With Acute Myocardial Infarction. Angiology. 2014. Online. DOl: 10.1177/0003319714542997

18. Dinerman JL, MehtaJL, Saldeen TGP, et al. Increased neutrophil elastaserelease in unstable angina pectoris and acutemyocardial infarction. Journal of the American College of Cardiology. 1990;15(7):1559-63.

19. Bian C, Wu Y, Shi Y. Predictive value of the relative lymphocyte count in coronary heart disease. Heart Vessels. 2010;25(6):469-73.

20. Meyer-SabellekW, Brasch H. Aterosclerosis, inflammation, leukocyte function and the effect of statins. J Hypertens. 2006;24(12):2349-51. 